\title{
Commentary: The enhanced recovery train is leaving the station-Compliance with phase of care guidelines is associated with earlier extubation and shorter length of stay as part of an enhanced recovery after cardiac surgery program
}

\author{
Subhasis Chatterjee, MD
}

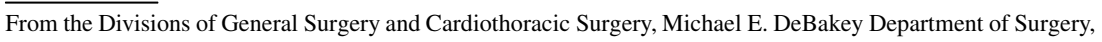
Baylor College of Medicine, Houston, Tex; and Department of Cardiovascular Surgery, Texas Heart Institute, Houston, Tex.

Disclosures: Author has nothing to disclose with regard to commercial support.

Received for publication May 29, 2019; accepted for publication May 31, 2019; available ahead of print July 10, 2019.

Address for reprints: Subhasis Chatterjee, MD, Baylor College of Medicine, One Baylor Plaza, MS: BCM 390, Houston, TX 77030-3411 (E-mail: subhasis.chatterjee@bcm.edu).

J Thorac Cardiovasc Surg 2020;159:1405-6

$0022-5223 / \$ 36.00$

Copyright (c) 2019 by The American Association for Thoracic Surgery

https://doi.org/10.1016/j.jtcvs.2019.05.067
}

In this issue of the Journal, Grant and colleagues ${ }^{1}$ from Johns Hopkins Hospital report on the efficacy of an enhanced recovery program (ERP) in cardiac surgery and insights into its implementation. Although the rationale behind ERPs is broadly shared, demonstrating tangible benefits increases the likelihood of wider adoption. The Hopkins experience builds on the ERP highlighted by Williams and associates ${ }^{2}$ and the recently published guidelines by Engelman and colleagues ${ }^{3}$ of the ERAS Cardiac group.

To focus on discrete interventions, the ERP protocol is divided into specific phases of care: preoperative, intraoperative, and postoperative. Grant and colleagues ${ }^{1}$ looked at process compliance with 7 discrete interventions in the preoperative and intraoperative care phases. By dividing the cohort into low- and high-compliance groups, they compared postoperative extubation and phase of care lengths of stay in each group. After exclusion of left ventricular assist device implants, transplants, and aortic surgical cases, the matched cohort yielded a low-risk group $(80 \%$ of whom underwent coronary artery bypass grafting) with a mean left ejection fraction of $54 \%$ and a Society of Thoracic Surgery predicted risk of mortality of $1.55 \%$.

Obviously, there was some intrinsic variability in process measure compliance, because decisions were deferred to the individual anesthesiologist (most importantly, the decision about reversal of neuromuscular blockade and administration of gabapentin). It is notable, however, that at the start of the experience the low (2/7) average compliance level had improved considerably (5/7). Indeed, the highcompliance group achieved a $48 \%$ intraoperative extubation rate.

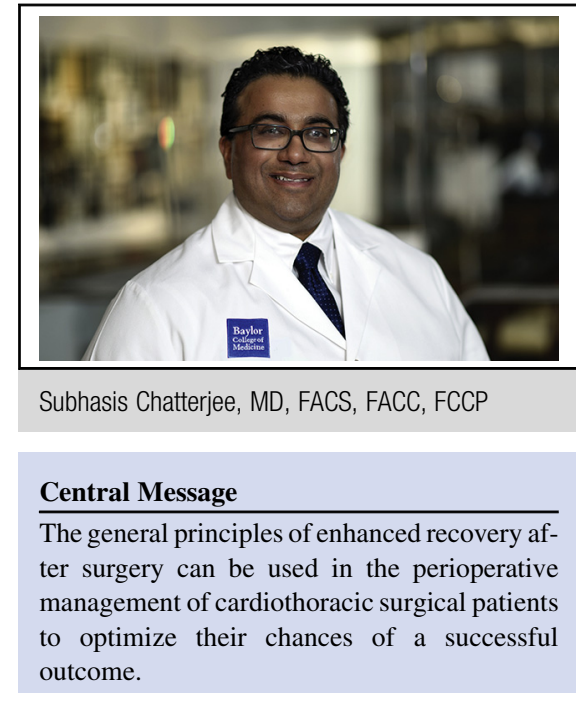

See Article page 1393.

In the 1980s and 1990s, cardiac surgery with high-dose opioid-based anesthesia resulted in routine intubation of patients 12 to 24 hours afterwards. Although an ERP is more than just "fast track by another name,"4 it does build on the "fast track" early extubation experience for comprehensive perioperative care. Earlier extubation in low- and mediumrisk patients undergoing coronary artery bypass grafting is now a class I (level of evidence B) recommendation in the American College of Cardiology and American Heart Association coronary artery bypass grafting guidelines. ${ }^{5}$ Indeed, extubation within 6 hours is a Society of Thoracic Surgeons quality indicator, despite suggestions that there may not be a meaningful clinical difference at the 12-hour versus the 6hour mark. ${ }^{6}$ For better or worse, this is how we are judged.

Grant and colleagues ${ }^{1}$ have pushed the envelope to the "final frontier" of early extubation in the operating room (OR) by embracing ERP principles. It is one thing, however, to have demonstrated that it can be done; the question remains, should it be done? Because rapid OR turnover is often necessary to avoid delaying the next case, it would be worthwhile to know whether there was a delay waiting for OR extubation as opposed to prompt intensive care 
unit transfer. Although this study did not report turnover time, the experience at the University of Pittsburgh described by Subramanian and associates ${ }^{7}$ suggests there may not be a delay. In a similar low-risk cohort of 1198 patients, they were able to extubate $30 \%$ in the OR, with only $1.4 \%$ requiring reintubation within 23 hours. It was notable that the OR extubation group actually spent 13 minutes less in the OR (373 minutes) than the intensive care unit extubation group (386 minutes), suggesting that prolonged OR time is unlikely.

Concerns with early extubation rightfully focus on a concern for premature extubation. Ensuring adequate analgesia; avoiding hypothermic shivering, which increases metabolic demand and respiratory distress; and the concerns for ensuring a period of hemodynamic instability and observation for bleeding are all reasons cited to avoid extubation in the OR. The experience of Grant and colleagues, ${ }^{1}$ however, suggests that OR extubation did not come at the expense of those concerns. The key was that there was no increase in reintubations; indeed; the intraoperative group had none. The rates of reintubation were $6 \%$ for the overall cohort and the low-compliance cohort, $5 \%$ for the high compliance cohort, and $0 \%$ for the intraoperative extubation cohort. This suggests that OR extubations did not come at the expense of reintubations. Ultimately, the metrics used to evaluate ERPs- to gauge whether they are worthwhile-include reductions in complications, length of stay, and costs.
The Johns Hopkins group ${ }^{1}$ are to be commended for creating a multidisciplinary ERP for cardiac surgery to help in the formulation and implementation of the processes. By demonstrating reductions in time to extubation and hospital length of stay, along with institutional commitment and champions across several specialties and members of the care team, successful ERP implementation can be achieved.

\section{References}

1. Grant MC, Isada T, Ruzankin P, Whitman G, Lawton JS, Dodd-o J, et al. Results from an enhanced recovery program for cardiac surgery. J Thorac Cardiovasc Surg. 2020;159:1393-402.e7.

2. Williams JB, McConnell G, Allender JE, Woltz P, Kane K, Smith PK, et al. Oneyear results from the first US-based enhanced recovery after cardiac surgery (ERAS Cardiac) program. J Thorac Cardiovasc Surg. 2019;157:1881-8.

3. Engelman DT, Ben Ali W, Williams JB, Perrault LP, Reddy VS, Arora RC, et al. Guidelines for perioperative care in cardiac surgery: Enhanced Recovery After Surgery Society recommendations. JAMA Surg. 2019;154:755-66.

4. Chatterjee S. Enhanced recovery after cardiac surgery: a game changer, passing fad, or somewhere in between? J Thorac Cardiovasc Surg. 2019;157: 1889-90.

5. Hillis LD, Smith PK, Anderson JL, Bittl JA, Bridges CR, Byrne JG, et al. 2011 ACCF/AHA guideline for coronary artery bypass graft surgery: executive summary: a report of the American College of Cardiology Foundation/American Heart Association task force on practice guidelines. Circulation. 2011;124: 2610-42. Erratum in: Circulation. 2011;124:e956. Erratum in: Circulation. 2012;126:e105.

6. Crawford TC, Magruder JT, Grimm JC, Sciortino C, Conte JV, Kim BS, et al. Early extubation: a proposed new metric. Semin Thorac Cardiovasc Surg. 2016;28: 290-9.

7. Subramaniam K, DeAndrade DS, Mandell DR, Althouse AD, Manmohan R, Esper SA, et al. Predictors of operating room extubation in adult cardiac surgery. J Thorac Cardiovasc Surg. 2017;154:1656-65.e2. 